\title{
Article
}

\section{Homeomorphic Arrangements of Smooth Manifolds}

\author{
Eran Liberman and Mina Teicher*
}

check for

updates

Citation: Liberman, E.; Teicher, M. Homeomorphic Arrangements of Smooth Manifolds. Symmetry 2021, 13, 981. https://doi.org/10.3390/ sym13060981

Academic Editor: Abraham A. Ungar

Received: 20 April 2021

Accepted: 21 May 2021

Published: 1 June 2021

Publisher's Note: MDPI stays neutral with regard to jurisdictional claims in published maps and institutional affiliations.

Copyright: (c) 2021 by the authors. Licensee MDPI, Basel, Switzerland. This article is an open access article distributed under the terms and conditions of the Creative Commons Attribution (CC BY) license (https:/ / creativecommons.org/licenses/by/ $4.0 /)$.
Department of Mathematics, Bar-Ilan University, Ramat Gan 5290002, Israel; liberme1@biu.ac.il

* Correspondence: teicher@math.biu.ac.il

\begin{abstract}
Symmetry between mathematical constructions is a very desired phenomena in mathematics in general, and in algebraic geometry in particular. For line arrangements, symmetry between topological characterizations and the combinatorics of the arrangement has often been studied, and the first counterexample where symmetry breaks is in dimension 13. In the first part of this paper, we shall prove that two arrangements of smooth compact manifolds of any dimension that are connected through smooth functions are homeomorphic. In the second part, we prove this in the affine case in dimension 4 .
\end{abstract}

Keywords: smooth manifold; lines; homeomorphism

\section{Introduction}

An arrangement is a finite collection of affine subspaces (of possibly varying dimensions) in $\mathbb{R}^{l}$ or $\mathbb{C}^{l}$, or a collection of linear subspaces of a projective space $\mathbb{C P}^{l-1}$ or $\mathbb{R} \mathbb{P}^{l-1}$. The topology of the complement of the union of the planes is of considerable interest. In 1989, Randell [1] proved a deep theorem, which shows that two arrangements have the same topology if they can be transferred from one to the other using a smooth one parameter family of arrangements. This resulted in the invention of a new invariant-the moduli space. Randell's study was very fruitful and resulted in many important theorems concerning symmetry rope. For instance, in [2] it was implemented for lines which in this case are a linear subspace of $\mathbb{C}^{2}$, for example, the solution in $\mathbb{C}^{2}$ to the equation $y=(5+i) x+2+4 i$. In [3,4], it was proven that the combinatorial structure determines the fundamental group of the complement for a six line arrangement. Using Van Kampen theorems [5] and the Moishezon-Teicher algorithm [6], it was extended to seven and eight lines $[7,8]$. Later, it was generalized to nine lines in [9], ten lines in [10] and eleven lines in [11].

In this paper, we are going to improve Randell's theorem and we move to diffeomorphisms of smooth manifolds in general, a contemporary topic (see, e.g., [12,13]). Whereas Randell talks about changing hyperplane arrangements through smooth families of hyperplanes, we are going to show that we can also transfer from one arrangement of smooth manifolds to another using smooth families of hyperplane arrangements. As a consequence, we show that we can transform arrangements of lines to other arrangements of lines through symmetry of arrangements to polynomials, families of polynomials of any degree. Since lines in the complex planes are homeomorphic to two dimensional sets in $\mathbb{R}^{4}$, our theorems will use that idea for another improvement of the theorem.

\section{Definitions and Notations}

The following theorems and definitions are well known.

Theorem 1 (Topological invariance of dimension [14]). A non-empty n-dimensional topological manifold cannot be homeomorphic to an m-dimensional manifold unless $m=n$. 
Definition 1 ([14]). For any smooth manifold $M$, we define an open submanifold of $M$ to be any open subset with the subspace topology and with the smooth charts obtained by restricting those of $M$.

Theorem 2 (Open sub-manifold [14]). Suppose $M$ is a smooth manifold. The embedded submanifolds of codimension 0 in $M$ are exactly the open submanifolds.

Proposition 1 ([14]). Suppose $M$ and $N$ are smooth manifolds with or without boundary, and $F: M \rightarrow N$ is an injective smooth immersion. If any of the following holds, then $F$ is a smooth embedding:

(a) $\quad F$ is an open or closed map;

(b) $F$ is a proper map;

(c) $M$ is compact;

(d) $\quad M$ has empty boundary and $\operatorname{dim} M=\operatorname{dim} N$.

Theorem 3 (Proper continuous maps are closed [14]). Suppose $X$ is a topological space and $Y$ is a locally compact Hausdorff space. Then every proper continuous map $F: X \rightarrow Y$ is closed.

Definition 2. A stratification of a manifold is a partitioning of the manifold into a finite collection of submanifolds $\{U\}$ (called the strata) so that the following frontier condition is satisfied: Whenever $U$ and $V$ are strata with $V \cap \operatorname{cl}(U) \neq \varnothing$, then $V \subset \operatorname{cl}(U)$.

Definition 3 ([14]). A stratification is called a Whitney stratification if it satisfies Whitney's condition (b): For all strata $U, V$, with $V \cap c l(U) \neq \varnothing$, and for all $x \in V$, whenever $x_{i}$ and $y_{j}$ are sequences in $V$ and $U$, respectively, with $x_{i} \neq y_{i}$ so that $x_{i}$ converges to $x$ and $y_{i}$ converges to $x$, so that the secants $\overline{x_{i} y_{i}}$ converge to $l \in \mathbb{R P}^{n-1}$ and so that $T_{y_{i}} U$ converges to $\tau$ in the Grassmannian of dimension $U$ planes in $\mathbb{R}^{n}$, then $l \subset \tau$.

Theorem 4 ([1]). Any stratification in which the closure of every stratum is a smooth submanifold is a Whitney stratification.

Theorem 5 (Thom's first isotopy theorem [15]). Let $f: M \rightarrow \mathbb{R}$ be a proper, smooth map which is a submersion on each stratum of a Whitney stratification of $M$. Then there is a stratum-preserving homeomorphism $h: M \rightarrow \mathbb{R} \times\left(f^{-1}(0) \cap M\right)$ which is smooth on each stratum and commutes with the projection to $R$. In particular, the fibers of $f$ are homeomorphic by a stratum-preserving homeomorphism.

\section{Stereographic Projection}

The following definitions and claims are well known: the function

$$
\varphi_{n}: S^{n} \backslash(0,0, \ldots, 1) \rightarrow \mathbb{R}^{n}
$$

defined by

$$
\varphi_{n}\left(x_{1}, \ldots, x_{n+1}\right)=\left(\frac{x_{1}}{1-x_{n+1}}, \ldots, \frac{x_{n}}{1-x_{n+1}}\right)
$$

is a homeomorphism and so is from

$$
\psi_{n}: i S^{n} \backslash(0,0, \ldots,-1)
$$

to $\mathbb{R}^{n}$ defined by

$$
\psi_{n}\left(x_{1}, \ldots, x_{n+1}\right)=\left(\frac{x_{1}}{1+x_{n+1}}, \ldots, \frac{x_{n}}{1+x_{n+1}}\right)
$$


The inverse functions are:

$$
\begin{gathered}
\varphi_{n}^{-1}\left(y_{1}, \ldots, y_{n}\right)=\left(\frac{2 y_{1}}{\sum_{i=1}^{n} y_{i}^{2}+1}, \ldots, \frac{2 y_{n}}{\sum_{i=1}^{n} y_{i}^{2}+1}, 1-\frac{2}{\sum_{i=1}^{n} y_{i}^{2}+1}\right) \\
\psi_{n}^{-1}\left(y_{1}, \ldots, y_{n}\right)=\left(\frac{2 y_{1}}{\sum_{i=1}^{n} y_{i}^{2}+1}, \ldots, \frac{2 y_{n}}{\sum_{i=1}^{n} y_{i}^{2}+1},-1+\frac{2}{\sum_{i=1}^{n} y_{i}^{2}+1}\right) .
\end{gathered}
$$

The composition defined from $\mathbb{R}^{n}$ minus the origin to itself is:

$$
\psi_{n} \circ \varphi_{n}^{-1}\left(y_{1}, \ldots, y_{n}\right)=\varphi \circ \psi^{-1}\left(y_{1}, \ldots, y_{n}\right)=\left(\frac{y_{1}}{\sum_{i=1}^{n} y_{i}^{2}}, \ldots, \frac{y_{n}}{\sum_{i=1}^{n} y_{i}^{2}}\right)
$$

In this paper we denote $p:=(0,0,1), \varphi:=\varphi_{2}, \psi:=\psi_{2}$.

\section{Topological and Geometric Aspects of Manifolds}

With the previous theorems in mind we prove the following.

Lemma 1. Let $A, B$ and $C_{1}, \ldots, C_{n}$ be manifolds such that $B$ is a closed submanifold of $A$ and $C_{1}, \ldots, C_{n}$ are closed submanifolds of $B$. Assume that for all $1 \leq i \leq n, \operatorname{dim}\left(C_{i}\right)<\operatorname{dim}(B)$. Then $\bigcup_{i=1}^{n} C_{i}$ is nowhere dense in the subspace topology of $B$ and in addition $C l\left(B \backslash \bigcup_{i=1}^{n} C_{i}\right)=B$.

Proof. First we prove that for any $1 \leq i \leq n, C_{i}$ is nowhere dense. Indeed, since $C_{i}$ is closed it is sufficient to show that it has an empty interior. Assume to the contrary that $S$ is an open set of $B$ contained in $C_{i}$, then from the definition of subspace topology it is an open set also in $C_{i}$ by Theorem 2 . We get that $S$ is a manifold of dimension equal to the dimension of $B$ and also a manifold with a dimension equal to $C_{i}$. The contradiction to Theorem 1 proves the statement. Since a finite union is nowhere dense so is $\bigcup_{i=1}^{n} C_{i}$ and since $1 \leq i \leq n C_{i}$ is closed then $C l\left(B \backslash \bigcup_{i=1}^{n} C_{i}\right)=B$

Proposition 2. Let $A, B, C, D$ be Hausdorff spaces where $A$ is compact. Let $f: A \times B \rightarrow C$ be a continuous function and $g: B \rightarrow D$ be continuous and proper. Let $h: A \times B \rightarrow C \times D$ be defined by sending $(x, y)$ to $(f(x, y), g(y))$ then $h$ is continuous and proper.

Proof. Let $p$ be the projection of $C \times D$ to $D$ and let $S$ be some set. Then, an element $(a, b)$ is in $h^{-1}(S)$ if there exist $(c, d) \in C \times D$ such that $h((a, b))=(c, d)$. By definition, $h((a, b)=(f(a, b), g(b))$ and $h((a, b))=(c, d)$ if and only if $(f(a, b), g(b))=(c, d))$. This implies that $f(a, b)=c$ and $g(b)=d$ so $h^{-1}(S)=f^{-1}(S) \cap g^{-1}(p(S)$. First we prove that $h$ is indeed continuous. Let $S_{1}$ be an open set since $p$ is an open function and $f, g$ are continuous. Then $f^{-1}(S) \cap g^{-1}(p(S)$ is an intersection of the two open sets and, therefore, open. Next we will prove that $h$ is proper: let $S$ be a compact set. In particular it is closed so $h^{-1}(S)$ is closed. On the other hand, $h^{-1}(S)=f^{-1}(S) \cap g^{-1}\left(p(S) \subset A \times g^{-1} p(S)\right.$ since $p$ is continuous, $P(S)$ is compact and since $h$ is proper $g^{-1} p(S)$ is also compact. Since $A$ and $g^{-1} p(S)$ are compact so is $A \times g^{-1} p(S)$. To conclude, we get that $h^{-1}(S)$ is closed and a subset of compact subset and, therefore, compact. 
Proposition 3. Let $Q$ and $M$ be smooth manifolds and $f: Q \times \mathbb{R} \rightarrow M$ a smooth function. For any $t \in \mathbb{R}$, let $f^{t}: Q \rightarrow M$ be the function sending $q$ to $f(q, t)$. Let $F: Q \times \mathbb{R} \rightarrow M \times \mathbb{R}$ be the function that sends $(g, t)$ to $(f(g, t), t)$. Then

1. if any $t \in \mathbb{R} f^{t}$ is injective then $F$ is injective;

2. if $f$ is smooth then $F$ is smooth;

3. If $f$ is smooth and for any $t \in \mathbb{R} f^{t}$ is an immersion then $F$ is an immersion;

4. if $f$ is smooth and $\operatorname{dim}(Q)=0$ then $F$ is an immersion;

5. if $Q$ is compact, $f$ is smooth, for any $t \in \mathbb{R} f^{t}$ is injective and if $\operatorname{dim}(Q)>0$ it is also an immersion then $F$ is an embedding with a closed image;

6. if $F$ is an embedding and $\pi$ is the projection of $M \times \mathbb{R}$ to the second factor then $\left.\pi\right|_{\operatorname{Im}(F)}$ is a submersion.

\section{Proof.}

1. Let $\left(q_{1}, t_{1}\right),\left(q_{2}, t 2\right) \in Q \times \mathbb{R}$ such that $F\left(\left(q_{1}, t_{1}\right)=F\left(q_{2}, t_{2}\right)\right.$. Then by the definition of $F$ we get that $\left(f\left(q_{1}, t_{1}\right), t_{1}\right)=\left(f\left(q_{2}, t_{2}\right), t_{2}\right)$. Therefore, $t_{1}=t_{2}$ and we denote it as $t$, so we get that $f\left(q_{1}, t\right)=f\left(q_{2}, t\right)$. By the definition of $f^{t}$ this implies that $f^{t}\left(q_{1}\right)=f^{t}\left(q_{2}\right)$ and since $f^{t}$ is injective, then $q_{1}=q_{2}$.

2. Since $f$ is a smooth function from $Q \times \mathbb{R}$ to $M$, there exist for every $p \in Q \times \mathbb{R}$ smooth charts $(U, \varphi)$ containing $p$ and $(V, \psi)$ containing $f(p)$ such that $f(U) \subset V$ and $\psi \circ f \circ \varphi^{-1}$ is a smooth function from $U$ to $V$. Since the domain is $Q \times \mathbb{R}$ then $(U, \varphi)=\left(U_{1} \times \mathbb{R}, \varphi_{1} \times I d_{\mathbb{R}}\right)$. Since $(V, \psi)$ is a chart of $M$ then $\left(V \times \mathbb{R}, \psi \times I d_{\mathbb{R}}\right)$ is a chart for $M \times \mathbb{R}$. If we take the point $q \in U$ it is equal to $\left(q_{1}, t\right)$ where $q_{1} \in U_{1}$ and $t \in \mathbb{R}$. We look at the charts $\left(U_{1} \times \mathbb{R}, \varphi \times I d_{\mathbb{R}}\right)$ and $\left(V \times I d_{\mathbb{R}}, \psi \times I d_{\mathbb{R}}\right)$. Then

$$
\left(\psi \times I d_{\mathbb{R}}\right) \circ F \circ\left(\varphi \times I d_{\mathbb{R}}\right)^{-1}\left(q_{1}, t\right)=\left(\psi \times I d_{\mathbb{R}}\right) \circ\left(f \times i d_{\mathbb{R}}\right) \circ\left(\varphi^{-1}\left(q_{1}\right), t\right)
$$

which is equal to

$$
\left(\psi \times I d_{\mathbb{R}}\right) \circ\left(f \circ \varphi^{-1}\left(q_{1}, t\right), t\right)=\left(\psi \circ f \circ \varphi^{-1}\left(q_{1}, t\right), t\right) .
$$

Let us assume that $M$ is of order $k$. Then $\psi \circ f \circ \varphi^{-1}$ have $k$ component functions $f_{1}, \ldots, f_{k}$ which are all smooth. So we can write $F$ as $k+1$ components $f_{1}, \ldots, f_{k}, p$ where $p$ is the projection from $Q \times \mathbb{R}$ to $\mathbb{R}$. We can see that all the components are smooth and, therefore, $F$ is smooth.

3. Let $p$ be a point as in the previous paragraph and that $\psi \times I d_{\mathbb{R}} \circ F \circ \varphi^{-1}$ has $\mathrm{k}$ +1 components $f_{1}, \ldots, f_{k}, t$. Since $\varphi=\varphi_{1} \times I d_{\mathbb{R}}$ then the coordinates of the domain of $\psi \times I d_{\mathbb{R}} \circ F \circ \varphi^{-1}$ are $x_{1}, \ldots, x_{m}, t$ when we assume that the order of $Q$ is $m$. Let $1 \leq i \leq k$ and $1 \leq j \leq m$, then the partial derivative of $f_{i}$ in the coordinate $x_{j}$ is equal to

$$
\lim _{h \rightarrow 0} \frac{\left.f_{i}\left(x_{1}, \ldots, x_{j-1}, x_{j}+h, x_{j+1}, \ldots, x_{k}, t\right)-f_{i}\left(x_{1}, \ldots x_{k}, t\right)\right)}{h}
$$

which is equal to

$$
\lim _{h \rightarrow 0} \frac{f_{i}^{t}\left(x_{1}, \ldots, x_{j-1}, x_{j}+h, x_{j+1}, \ldots, x_{k}\right)-f_{i}^{t}\left(\left(x_{1}, \ldots x_{k}, t\right)\right)}{h}
$$

where $f_{i}^{t}$ is the $i$-th component of $\psi \circ f^{t} \circ \varphi_{1}^{-1}$. We can see that the partial derivative of $f_{i}$ in the coordinate $x_{j}$ is equal to the partial derivative of $f_{i}^{t}$ in the coordinate 
$x_{j}$. The $x_{j}$ partial derivative of $t$ is 0 and the $t$-th derivative of $t$ is 1 . To conclude, the Jacobian of $F$ in the point $p$ is

$$
\left[\begin{array}{cccc} 
& \mu & 0 \\
& & & \\
* & & \\
* & \cdots & * & 1
\end{array}\right]
$$

where $A$ is the Jacobian of $f^{t}$. Since $f^{t}$ is an immersion so is $F$.

4. Since $\operatorname{dim}(Q)=0$, it is a union of points with the discrete topology and every point has a homeomorphism to $\mathbb{R}^{0}$ which is precisely one point by a function that sends one point to the other. So if we take one point $a \times \mathbb{R}$ and the homeomorphism to $\mathbb{R}$ as the natural one, then we have that one function $f(t)=z$ where $z$ is the variable of $\mathbb{R}$ in $M \times \mathbb{R}$ which is obviously an immersion.

5. By paragraphs (1)-(4) $F$ is injective immersion. Since the identity is obviously proper, then by Lemma $3 F$ is proper. So by Proposition 1 it is a smooth embedding. By Theorem 3 it is closed; in particular, it has a closed image.

6. It is sufficient to prove that $F \circ \pi$ is a submersion but $F \circ \pi$ is a projection of the second factor of $Q \times \mathbb{R}$ which is known to be a submersion.

\section{Main Theorem}

In order to prove the main Theorem 4, we need the following lemma.

Lemma 2. Let $M, S$ be smooth manifolds. $H$ is a finite set of closed smooth submanifolds of $M$. $s: M \rightarrow S$ is a submersion such that:

1. for every $h \in H, \operatorname{dim}(h)<\operatorname{dim}(M)$;

2. for every $h_{1}, h_{2} \in H$ such that $h_{1} \subsetneq h_{2}, \operatorname{dim}\left(h_{1}\right)<\operatorname{dim}\left(h_{2}\right)$;

3. for every $h_{1}, h_{2} \in H$, there exist $H_{1} \subset H$ such that $h_{1} \cap h_{2}=\cup H_{1}$. For all $h \in H$, $\left.s\right|_{h}$ is a submersion.

Let us denote

1. for $h \in H \bar{h}=\left\{h \backslash \bigcup_{h_{1} \in H \wedge h_{1} \subsetneq h} h_{1} \mid h \in H\right\}$;

2. $\bar{H}:=\bigcup_{h \in H}\{\bar{h}\} ;$

3. $\bar{M}=M \backslash(\cup \bar{H})$;

4. $W:=\bar{H} \cap\{\bar{M}\}$.

Then $\bar{H} \cup M \backslash \cap \bar{H}$ is Whitney's stratification, and for all $w \in W,\left.s\right|_{w}$ is a submersion.

Proof. Since every element in $\bar{H}$ is a submanifold minus a finite union of closed sets, it is an open submanifold of $h$ and by Theorem 2 every $\bar{h} \in \bar{H}$ is a submanifold. It is also closed in $M$ and, therefore, $\bar{M}$ is also an open submanifold. It is obvious that $\cup W=M$. Next we will prove that every two different sets in $W$ are disjoint. It is sufficient to show for elements in $\bar{H}$. Let $\overline{h_{1}}, \overline{h_{2}} \in \bar{H}$ where $\overline{h_{1}} \neq \overline{h_{2}}$. Assume to the contrary that $x \in \overline{h_{1}} \cap \overline{h_{2}}$. From the definition of $\bar{H}$ there exist $h_{1}, h_{2} \in H$ such that $\overline{h_{1}}=h_{1} \backslash \bigcup_{h \in H \wedge h \subseteq h_{1}} h$ and $\overline{h_{2}}=h_{2} \backslash \bigcup_{h \in H \wedge h \subset h_{2}} h$. We can see that $x \in h_{1}$ and $x \in h_{2}$ and, therefore, $x \in h_{1} \cap h_{2}$. By our assumption there exist $H_{1} \subset H$ such that $h_{1} \cap h_{2}=\cup H_{1}$. Therefore, $x \in \cup H_{1}$ which implies that there exist $h_{3} \in H_{1}$ such that $x \in h_{3}$ and $h_{3} \subset h_{1} \cap h_{2}$. If $h_{1}$ were equal to $h_{2}$ then $w_{1}$ would be equal to $w_{2}$. Therefore, there exist $h_{i}(i=1,2)$ such that $h_{1} \cap h_{2} \subsetneq h_{i}$, hence $h_{3} \subsetneq h_{i}$ and, therefore, $x \notin w_{i}$. This contradiction proves our statement.

Until now we proved that $\bar{W}$ is a partitioning of the manifold $M$ into a finite collection of submanifolds. Let $w \in W$. Then it is equal to $h_{1} \backslash \underset{h \in H \wedge h \subsetneq h_{1}}{\bigcup} h$ since by our assumption 
every $h$ that is strictly contained in $h_{1}$ has a smaller dimension by Lemma $1 C l(w)=h_{1}$. Similarly, since for every $h \in H \operatorname{dim}(h)<\operatorname{dim}(M)$ and $\bar{M}=M \backslash \cup H$. Then by Lemma 1 $C l(\bar{M})=M$. Assume now that we have two elements $w_{1}, w_{2}$ such that $C l\left(w_{1}\right) \cap w_{2} \neq \varnothing$. Then, by our last statement, $C l\left(w_{1}\right)=h_{1}$ for some $h_{1} \in H$ and $w_{2}=h_{2} \backslash \bigcup_{h \in H \wedge h \subsetneq h_{2}} h$ for $h_{2} \in H$. If $h_{1} \cap h_{2} \neq h_{2}, h_{1} \cap h_{2} \subsetneq h_{2}$, and, therefore, $h_{1} \cap h_{2} \cap w_{2}=\varnothing$. Since $w_{2} \subseteq h_{2}$ we get that $h_{2} \cap w_{2}=w_{2}$ and, therefore, $h_{1} \cap w_{2}=\varnothing$. This contradiction forces us to say that $h_{1} \cap h_{2}=h_{2}$ which implies that $h_{2} \subseteq h_{1}$ and, therefore, $w_{2} \subseteq h_{1}$. Since $C l(\bar{M})=M$, it is obvious that every element in $W$ is a subset of $c l(\bar{M})$. To conclude, we get that $\bar{W}$ is a stratification. Since for every $w \in \bar{W}$ there is $h \in H$ such that $C l(w)=h$ and every $h \in H$ is a smooth submanifold and the closure of $\bar{M}=M$, which is also a smooth manifold, by Theorem $4 \bar{W}$ is also a Whitney stratification. Since submersion is a local property and $s \mid h$ and are submersions, this is also true for their open subspaces, namely the elements in $\bar{W}$.

Proposition 4. Let $M$ be a compact smooth manifold, $J$ a finite set. For every $j \in J, Q_{j}$ is a compact smooth manifold and $g_{j}: Q_{j} \times \mathbb{R} \rightarrow M$ is a smooth function. For every $j \in J$ and $t \in \mathbb{R}$, let $g_{j}^{t}: Q_{j} \rightarrow M$ be the function that sends $q$ to $g_{j}(q, t) . f: J \times J \rightarrow P(J)$ is a function such that:

1. for any $(i, j) \in J \times J$ and for all $t \in \mathbb{R} \operatorname{Im}\left(g_{i}^{t}\right) \cap \operatorname{Im}\left(g_{j}^{t}\right)=\underset{k \in f((i, j))}{\bigcup} \operatorname{Im}\left(g_{k}^{t}\right)$;

2. for all $j \in J$ and $t \in \mathbb{R} g_{j}^{t}$ is injective;

3. for all $j \in J$ and $t \in \mathbb{R}$ if $\operatorname{dim}\left(Q_{j}\right)>0 g_{t}^{j}$ is an immersion;

4. for all $j \in J \operatorname{dim}\left(Q_{j}\right)<\operatorname{dim}(M)$;

5. for all $j, k \in J$ if there exist $t \in \mathbb{R}$ such that $\operatorname{Im}\left(g_{t}^{k}\right) \subsetneq \operatorname{Im}\left(g_{j}^{t}\right)$ then $\operatorname{dim}\left(Q_{k}\right)<\operatorname{dim}\left(Q_{j}\right)$.

Then $M \backslash \bigcup_{j \in J} \operatorname{Im}\left(g_{j}^{0}\right)$ is homeomorphic to $M \backslash \bigcup_{j \in J} \operatorname{Im}\left(g_{j}^{1}\right)$.

Proof. We define for any $j \in J, G_{j}: Q_{j} \times \mathbb{R} \rightarrow M \times \mathbb{R}$ by $G_{j}(x, t):=\left(g_{j}^{t}(x), t\right)$. Since $g_{j}$ is smooth, $Q_{j}$ is compact. For all $t \in \mathbb{R}, g_{j}^{t}$ is injective and if $\operatorname{dim}\left(Q_{j}\right)>0$ then it is also an immersion. Then, by Proposition $3 G_{j}$ is an embedding with a closed image, and for $\pi$ equal to the projection of $M \times \mathbb{R}$ to the second factor $\left.\pi\right|_{I m\left(G_{j}\right)}$ is a submersion.

Now we are going to show that for any $(i, j) \in J \times J$,

$$
\operatorname{Im}\left(G_{i}\right) \cap \operatorname{Im}\left(G_{j}\right)=\bigcup_{k \in f((i, j))} \operatorname{Im}\left(G_{k}\right)
$$

Indeed, $(x, t) \in \operatorname{Im}\left(G_{i}\right)$ if and only if $x \in g_{t}^{t}$. Therefore, $(x, t) \in \operatorname{Im}\left(G_{i}\right) \cap \operatorname{Im}\left(G_{j}\right)$ if and only if $x \in \operatorname{Im}\left(g_{i}^{t}\right) \cap \operatorname{Im}\left(g_{j}^{t}\right)$ which by (1) this happens if and only if $x \in \bigcup_{i \in f(k)} \operatorname{Im}\left(G_{i}^{t}\right)$ and happens if and only if $(x, t) \in \bigcup_{k \in f((i, j))} \operatorname{Im}\left(G_{k}\right)$. Let $i, j \in J$. If $\operatorname{Im}\left(G_{i}\right) \subsetneq \operatorname{Im}\left(G_{j}\right)$. We would like to show that $\operatorname{dim}\left(\operatorname{Im}\left(G_{i}\right)\right)<\operatorname{dim}\left(\operatorname{Im}\left(G_{i}\right)\right)$. We know that $(x, t)$ is in $\operatorname{Im}\left(G_{i}\right)$ if and only if $x \in g_{j}^{t}$ and the same is true for $j$. So if $(x, t) \in \operatorname{Im}\left(G_{j}\right) \backslash \operatorname{Im}\left(G_{i}\right)$, then $x \in \operatorname{Im}\left(g_{i}^{t}\right) \backslash \operatorname{Im}\left(g_{j}^{t}\right)$ and for this specific $t$ if $x \in g_{i}^{t}$ then $(x, t) \in \operatorname{Im}\left(G_{i}\right)$ which implies that $(x, t) \in \operatorname{Im}\left(G_{i}\right)$ and, therefore, $x \in g_{t}^{t}$. Combining these two facts, we get that $\operatorname{Im}\left(g_{i}^{t}\right) \subsetneq \operatorname{Im}\left(g_{j}^{t}\right)$ which implies by our condition that $\operatorname{dim}\left(Q_{j}\right)<\operatorname{dim}\left(Q_{l}\right)$ which imply that $\operatorname{dim}\left(Q_{j} \times \mathbb{R}\right)<\operatorname{dim}\left(Q_{l} \times \mathbb{R}\right)$ which means that $\operatorname{dim}\left(\operatorname{Im}\left(G_{j}\right)\right)<\operatorname{dim}\left(\operatorname{Im}\left(G_{l}\right)\right)$, as needed. Let $H:=\left\langle\operatorname{Im}\left(G_{j}\right) \mid j \in J\right\rangle$. Then $H$ is a finite subset of closed smooth manifolds. For all $h_{1}, h_{2}$, there exist $H_{1}$ such that $h_{1} \cap h_{2}=\cup H$, for all $h_{1}, h_{2} \in H$ such that $h_{1} \subsetneq h_{2} \operatorname{dim}\left(h_{1}\right)<\operatorname{dim}\left(h_{2}\right)$ and for all $h \in H$ $\operatorname{dim}(h)<\operatorname{dim}(N)$.

We denote $W=\left\{h \backslash \bigcup_{h_{1} H \wedge h_{1} \subsetneq h} \mid h \in H\right\}, N:=(M \times \mathbb{R}) \backslash \cup H$ and $\bar{W}:=W \cup\{N\}$. Then by Lemma $2 \bar{W}$ is a Whitney stratification and for all $w \in \bar{W},\left.\pi\right|_{w}$ is a submersion, and since $\mathrm{M}$ is compact. $\pi$ is proper on $M \times \mathbb{R}$. Therefore, by Theorem 5 , there is a 
homeomorphism from $\pi^{-1}(0)$ to $\pi^{-1}(1)$ which is a stratum-preserving homeomorphism, so we can see that $\pi^{-1}(0)=M$. If $h \in \bar{H}$ then there exist $k \in P(I) \backslash \varnothing$ such that $h=H(k)$ $\pi^{-1}(1) \cap h=\bigcap_{i \in k} h_{1}^{i} \subset h_{1}^{l}$ where $l$ is some element in $k$. In particular, for $i \in I$, if $h=H(\{i\})$, $\left.\pi^{-1}(0) \cap h=h_{1}^{i}\right)$, so $\pi^{-1}(0) \cup \bar{H}=\bigcup_{i \in I} h_{1}^{i}$ which means that $\pi^{-1}(1) \cap \bar{N}=M \backslash \bigcup_{i \in I} h_{1}^{i}$. In the same way, $\pi^{-1}(0) \cap \bar{N}=M \backslash \bigcup_{i \in I} h_{0}^{i}$ and, therefore, they are homeomorphic.

\section{Adaptation of the Main Theorem to Curves}

This section is a corollary of the main theorem in the case of curves. We are going to use the following definition for simplification.

Definition 4. Let $F: X \times \mathbb{R} \rightarrow Y$ be a function. We define for every $t \in \mathbb{R}, F_{t}: X \rightarrow Y$ by $F_{t}(x)=F(x, t)$. Then we say that $F$ satisfies condition 1 if it satisfies the following conditions:

1. F is smooth;

2. for every $t \in \mathbb{R}, F_{t}$ is injective;

3. if $\operatorname{dim}(X)>0$ then for every $t \in \mathbb{R}, F_{t}$ is injective.

Theorem 6. Let $F: S^{2} \times \mathbb{R} \rightarrow S^{2} \times S^{2}$ be a function such that $F$ on $S^{2} \backslash p$ is $(\varphi \times I d) \circ g \circ$ $\left(\varphi^{-1} \times \varphi\right)^{-1}$ where $g(x, y, t)=\left((x, y),\left(H_{1}, H_{2}+\left(x^{2}+y^{2}\right)^{k}\right)\right.$, such that $H_{1}, H_{2}$ are polynomials of the variables $x, y$ over the smooth function with one variable, $\left.k=\max \left\{\operatorname{deg}\left(H_{1}\right), \operatorname{deg}\left(H_{2}\right)\right)\right\}$ and $(p, t)$ sends to $p \times p$. Then $F$ satisfies condition 1 .

Proof. Let

$$
\begin{aligned}
& H_{1}:=\sum_{i, j} h_{i j}^{1} x^{i} y^{j}, \\
& H_{2}:=\sum_{i, j} h_{i j}^{2} x^{i} y^{j}
\end{aligned}
$$

such that for all $i, j$ and $k=1,2, h_{i j}^{k}$ is a smooth function. First, we prove that $F$ is a smooth function. Let $a \in S^{2} \backslash p$, then we choose for the domain the chart $\varphi_{1} \times I d$ and for the image $\varphi \times \varphi$, so we need to prove that

$$
(\varphi \times I d)^{-1} \circ F \circ(\varphi \times \varphi)
$$

is smooth. Indeed we get that

$$
(\varphi \times I d)^{-1} \circ F \circ(\varphi \times \varphi)=(\varphi \times I d)^{-1} \circ(\varphi \times I d) \circ g \circ(\varphi \times \varphi)^{-1} \circ(\varphi \times \varphi)=g .
$$

It is easy to see that " $g$ " is smooth. For the points $(p, t)$, we take the domain chart to be $\psi_{2} \times I d$ and the image chart to be $\psi_{2} \times \psi_{2}$. So we need to prove that $\left(\psi_{2} \times I d\right)^{-1} \circ F \circ$ $\left(\psi_{2} \times \psi_{2}\right)$ is smooth. Indeed for a point $(a, t)$ where $a \neq p$

$$
\begin{gathered}
\left(\psi_{2} \times I d\right)^{-} 1 \circ F \circ\left(\psi_{2} \times \psi_{2}\right)=\left(\psi_{2} \times I d\right)^{-} 1 \circ(\varphi \times I d) \circ g \circ\left(\varphi^{-1} \times \varphi\right)^{-1} \circ\left(\psi_{2} \times \psi_{2}\right) \\
=\left(\left(\psi_{2}\right)^{-} 1 \circ(\varphi) \times I d\right) \circ g \circ\left(\varphi^{-1} \circ \psi_{2} \times\left(\varphi^{-1} \circ \psi_{2}\right)\right) .
\end{gathered}
$$

Thus, $\psi_{2}$ is injective and $\psi_{2}\left(p_{1}\right)=(0,0)$. So if $(x, t) \in \mathbb{R}^{2} \backslash(0,0) \times \mathbb{R}$ and we denote $z:=x^{2}+y^{2}$, we get that $\left(\left(\psi_{1}\right)^{-} 1 \circ\left(\varphi_{1}\right) \times I d\right)(x, y, t)=\left(\frac{x}{z}, \frac{y}{z}, t\right)$. If we apply $g$ on the result we get:

$$
\left(\frac{x}{z}, \frac{y}{z}\right),\left(\sum_{i, j} h_{i j}^{1}(t) \frac{x^{i} y^{j}}{z^{i+j}}, \sum_{i, j} h_{i j}^{2} \frac{(x)^{i}(y)^{j}}{z^{i+j}}+\left(\left(\frac{(x)^{2}}{z^{2}}+\frac{(y)^{2}}{z^{2}}\right)^{k}\right)\right) .
$$


$\operatorname{Now}\left(\frac{x}{z}\right)^{2}+\left(\frac{y}{z}\right)^{2}=\frac{x^{2}+y^{2}}{z^{2}}=\frac{z}{z^{2}}=\frac{1}{z}$, so we get

$$
\left(\frac{x}{z}, \frac{y}{z}\right),\left(\sum_{i, j} h_{i j}^{1}(t) \frac{(x)^{i}(y)^{j}}{z^{i+j}}, \sum_{i, j} h_{i j}^{2} \frac{(x)^{i}(y)^{j}}{z^{i+j}}+\left(\left(\frac{1}{z}\right)^{k}\right)\right) .
$$

This is equal to

$$
\left(\frac{x}{z}, \frac{y}{z}\right),\left(\sum_{i, j} h_{i j}^{1}(t) \frac{(x)^{i}(y)^{j} z^{k-i-j}}{z^{k}}, \sum_{i, j} h_{i j}^{2} \frac{x^{i} y^{j} z^{k-i-j}}{z^{k}}+\left(\frac{1}{z}\right)^{k}\right) .
$$

If we denote $A:=\sum_{i, j} h_{i j}^{1} x^{i} y^{j} z^{k-i-j}$ and $B:=\sum_{i, j} h_{i j}^{2} x^{i} y^{j} z^{k-i-j}$, we note that since $k$ is always larger than $i+j$ in every summand, then when $(x, y)$ approaches $(0,0)$ then $A$ and $B$ approach 0 . So we get $\left(\frac{x}{z}, \frac{y}{z}\right),\left(\frac{A}{z^{k}}, \frac{B+1}{z^{k}}\right)$. If we apply $\left(\varphi^{-1} \circ \psi_{2}\right) \times\left(\varphi^{-1} \circ \psi_{2}\right)$, we get

$$
\left(\frac{\frac{x}{z}}{\left(\frac{x}{z}\right)^{2}+\left(\frac{y}{z}\right)^{2}}, \frac{\frac{y}{z}}{\left(\frac{x}{z}\right)^{2}+\left(\frac{y}{z}\right)^{2}}\right),\left(\frac{\frac{A}{z^{k}}}{\left(\frac{A}{z^{k}}\right)^{2}+\left(\frac{B+1}{z^{k}}\right)^{2}}, \frac{\frac{B+1}{z^{k}}}{\left(\frac{A}{z^{k}}\right)^{2}+\left(\frac{B+1}{z^{k}}\right)^{2}}\right) .
$$

This is equal to $(x, y),\left(\frac{A z^{k}}{A^{2}+(B+1)^{2}}, \frac{(B+1) z^{k}}{A^{2}+(B+1)^{2}}\right)$.

We can see that when $x, y$ converges to $(0,0)$ the expression converges to $(0,0),(0,0)$. If we apply $\left(\psi_{2} \times I d\right)^{-1} \circ F \circ\left(\psi_{2} \times \psi_{2}\right)$ on $(0,0, t)$, we get $((0,0),(0,0)$ because applying $\left(\psi_{2} \times I d\right)^{-1}$ will give us $(p, t)$ Then, applying $F$ will give us $p \times p$ and, finally, applying $\psi_{2} \times \psi_{2}$ will give us $((0,0),(0,0))$. So the function is continuous and a smooth function divided by a smooth function other than 0 in a small neighborhood is smooth. Let $t \in \mathbb{R}$. We will show that $F_{t}$ is injective and is an immersion for all $t \in \mathbb{R}$. First we show it is injective for an element in $S^{2} \backslash p$. Let $R:=S^{2} \backslash p \times t$ then $F=\left.(\varphi \times I d)\right|_{R} \circ g \circ(\varphi \times \varphi)$. Since $\left.\left(\varphi_{1} \times I d\right)\right|_{R}, g, \varphi \times \varphi$ are injective, $F_{t}$ is injective on $S^{2} \backslash p$. Since the $p \times p$ is not in the image of $\varphi \times \varphi$ then $S^{1} \backslash p_{1}$ is not going to $p \times p$ since $p_{1}$ is going to $p \times p$, then $F_{t}$ is injective. Now we will show that the function is an immersion. For a point $a \in S^{2} \backslash p$, we choose for the domain the chart $\alpha$ sending $X$ to $(\varphi(X), t)$ and for the image $\varphi \times \varphi$ we get $\alpha \circ F \circ \varphi \times \varphi=\alpha \circ(\varphi \times I d) \circ g \circ(\varphi \times \varphi)^{-1} \circ(\varphi \times \varphi)$. This function sends $(x, y)$ to $((x, y),(*, *))$. If we look at the minor of the Jacobian, we get

$$
\left(\begin{array}{ll}
a_{11} & a_{12} \\
a_{11} & a_{12}
\end{array}\right)=\left(\begin{array}{ll}
1 & 0 \\
0 & 1
\end{array}\right)
$$

so these points have rank 2. For the point " $p$ " we choose for the domain the chart $\beta$ sending $X$ to $(\psi(X), t)$. For the image $(\psi \times \psi)$ for similar reasons as before, the image is $((x, y),(*, *))$ which is also of rank 2 . Therefore, on these points the function has rank 2 , hence it is an immersion.

The next theorem is an adaptation to lines in $\mathbb{C}^{2}$ which are topologically equivalent to sets in $\mathbb{R}^{4}$.

Theorem 7. Let $g_{i}: \mathbb{R} \rightarrow P\left(\mathbb{R}^{4}\right)(i=1 . . n)$ be functions defined by $t \rightarrow\left\{\left(x, y, H_{1}, H_{2}\right) \mid x, y \in\right.$ $\mathbb{R}\}$ such that $H_{1}, H_{2}$ are polynomials of the variables $x, y$ over the smooth function with one variable. Let $\kappa_{i}: \mathbb{R} \rightarrow \mathbb{R}^{4}$ (for $\left.i=1 . . m\right)$ be a smooth function and let $f:[1 . . n] \times[1 . . n] \rightarrow[1 . . m]$ be a function such that the following conditions hold:

1. for all $t \in \mathbb{R}$ and $1 \leq i, j \leq n, g_{i}(t) \cap g_{j}(t)=\kappa_{f(i, j)}(t)$;

2. for all $i \neq j$ and $t \in \mathbb{R} \kappa_{i}(t) \cap \kappa_{j}(t)=\varnothing$;

3. if there exist $t \in \mathbb{R}$ and $i, r$ such that $\kappa_{i}(t) \in g_{r}(t)$ then there exists $j$ such that $f(i, j)=r$. 
Then

$$
R^{4} \backslash\left(\bigcup_{i=1}^{n} \operatorname{Im}\left(g_{i}(0)\right) \cup \bigcup_{i=1}^{m} \operatorname{Im}\left(\kappa_{i}(0)\right)\right)
$$

is homeomorphic to

$$
R^{4} \backslash\left(\bigcup_{i=1}^{n} \operatorname{Im}\left(g_{i}(1)\right) \cup \bigcup_{i=1}^{m} \operatorname{Im}\left(\kappa_{i}(0)\right)\right)
$$

Proof. Let $\phi: \mathbb{R}^{4} \rightarrow \mathbb{R}^{4}$ be the homeomorphism sending $(x, y, z, w)$ to $\left(x, y, z, w+\left(x^{2}+\right.\right.$ $\left.\left.y^{2}\right)^{k}\right)$ where $k=\max \left(\operatorname{deg}\left(H_{1}\right), \operatorname{deg}\left(H_{2}\right)\right)$. Let $J_{1}:=\left\{a_{i} \mid 1 \leq i \leq n\right\}, J_{2}:=\left\{b_{1}, b_{2}\right\}$, $J_{3}:=\left\{c, d_{i} \mid 1 \leq i \leq m\right\}$ and $J:=\bigcup_{i=1}^{3} J_{i}$. To $a_{i}$ we attach the manifold $S^{2}$ and the function $G_{i}: S^{2} \times \mathbb{R} \rightarrow S^{2} \times S^{2}$ which we defined piecewise on $S^{2} \backslash p$. It will be defined as $(\varphi \times I d) \circ(\varphi \times I d) \circ g_{i} \circ \phi \circ(\varphi \times \varphi)^{-1}$ and $p \times \mathbb{R}$ will be sent to $p \times p$. By Theorem 6 they satisfy condition (1). For $b_{i}$ we attach the manifold $S^{2}$ and the functions $\beta_{i}: S^{2} \times \mathbb{R} \rightarrow$ $S^{2} \times S^{2}$ are defined as follows: $\beta_{1}(a, t)=(a, p)$ and $\beta_{2}(a, t)=(p, a)$. It is easy to see that these functions satisfy condition (1). For $c$ and $d_{i}$ we attach a manifold with a single point which we denote by $P t$. We define the following function: for $c$ we define the constant function $P$ which sends every element to $p \times p$ and for $d_{i}$ we define the function $D_{i}: \mathbb{R} \rightarrow S^{2} \times S^{2}$ which is defined as $\kappa_{i} \circ \varphi^{-1} \times \varphi^{-1}$. Next we define $\tilde{f}: J \times J \rightarrow P(J)$ as follows:

1. $\tilde{f}\left(a_{i}, a_{j}\right)=\left\{c, f\left(a_{i}, a_{j}\right)\right\}$;

2. $\tilde{f}\left(a_{i}, b_{j}\right)=c$;

3. $\tilde{f}\left(a_{i}, c\right)=c$;

4. $\tilde{f}\left(a_{i}, d_{r}\right)= \begin{cases}d_{r} & \exists j \text { s.t. } f(i, j)=r \\ \varnothing & \text { otherwise }\end{cases}$

5. $\tilde{f}\left(b_{1}, b_{2}\right)=c$;

6. $\tilde{f}\left(b_{i}, c\right)=c$;

7. $\tilde{f}\left(b_{i}, d_{j}\right)=\varnothing$;

8. $\tilde{f}\left(c, d_{i}\right)=\varnothing$;

9. $\tilde{f}\left(d_{i}, d_{j}\right)=\varnothing$.

To complete the definition, we add that $\tilde{f}(X, X):=X$ and if $\tilde{f}(Y, X)$ is defined then $\tilde{f}(X, Y)=\tilde{f}(Y, X)$. Now we would like to prove that for any $(i, j) \in J \times J$ and for any $t \in \mathbb{R}$ if we denote the function attached to $k$ as $r_{k}$ we get that $r_{i}^{t} \cap r_{j}^{t}=\underset{k \in f((i, j))}{\bigcup} r_{k}^{t}$.

1. For every $k$ and $t \in \mathbb{R}$ the image of $G_{k}^{t}$ is equal to $G_{k}\left(S^{2} \times t\right)$, the image of $p \times t$ is $p \times p$ and the image of $S^{2} \backslash p \times t$ is equal to $(\varphi \times I d) \circ(\phi \times I d) g_{k} \circ(\varphi \times \varphi)^{-1}\left(S^{2} \backslash p \times t\right)$. Since $\varphi$ is surjective on $\mathbb{R}^{2}$, this is equal to $\phi \times I D\left(g_{k} \circ(\varphi \times \varphi)^{-1}\left(\mathbb{R}^{2} \times t\right)\right)$. So the intersection of the images of $G_{i}^{t}$ and $G_{j}^{t}$ is equal to a $p \times p$ union with

$$
(\phi \times I d) \circ g_{i} \circ(\varphi \times \varphi)^{-1}\left(\mathbb{R}^{2} \times t\right) \cap(\phi \times I d) \circ g_{j} \circ(\varphi \times \varphi)^{-1}\left(\mathbb{R}^{2} \times t\right)
$$

which is equal

$$
(\varphi \times \varphi)^{-1}\left((\phi \times I d) \circ g_{i}\left(\mathbb{R}^{2} \times t\right)\right) \cap(\varphi \times \varphi)^{-1}\left((\phi \times I d) \circ\left(g_{j}\right)\left(\mathbb{R}^{2} \times t\right)\right) .
$$

Since $(\varphi \times \varphi)^{-1}$ and $(\phi \times I d)$ are injective, this is equal to $(\varphi \times \varphi)^{-1} \circ(\phi \times I d)\left(\left(g_{i}\left(\mathbb{R}^{2} \times\right.\right.\right.$ $\left.t)) \cap\left(g_{j}\left(\mathbb{R}^{2} \times t\right)\right)\right)$. From our assumption, we find that $\left.g_{i}\left(\mathbb{R}^{2} \times t\right)\right) \cap\left(g_{j}\left(\mathbb{R}^{2} \times t\right)\right)=$ $\kappa_{f}(i, j)(t)$. So applying $(\varphi \times \varphi)^{-1} \circ(\phi \times I d)$ will give us $(\varphi \times \varphi)^{-1} \circ(\phi \times I d)\left(\kappa_{f(i, j)}(t)\right)$ as needed. 
2. As in paragraph (1) $\operatorname{Im}\left(G_{i}^{t}\right)=p \times p \cup(\varphi \times \varphi)^{-1} \circ(\phi \times I d) \circ g_{k} \circ(\varphi \times \varphi)^{-1}\left(\mathbb{R}^{2} \times t\right)$. Since $p \times p$ is also in $b_{j}$ and the image of $\varphi \times \varphi$ is $S^{2} \backslash p \times S^{2} \backslash p$, the intersection is precisely $p \times p$.

3. As in paragraph (1) $\operatorname{Im}\left(G_{i}^{t}\right)=p \times p \cup g_{k} \circ(\varphi \times \varphi)^{-1}\left(\mathbb{R}^{2} \times t\right)$. So the intersection with $p \times p$ is $p \times p$.

4. First, if there exists $j$ we already proved that $\left(G_{i}(t)\right) \cap\left(G_{j}(t)\right)=\kappa_{r}(t)$ and $\kappa_{r}(t)$ contain only one point, then $G_{i}(t) \cap \kappa_{r}(t)=\kappa_{r}(t)$. Next if $j$ do not exist then as in paragraph (1) $\operatorname{Im}\left(G_{i}^{t}\right)=p \times p \cup(\varphi \times \varphi)^{-1} \circ(\phi \times \operatorname{Id}) \circ g_{k} \circ(\varphi \times \varphi)^{-1}\left(\mathbb{R}^{2} \times t\right)$ and $\operatorname{Im}\left(D_{i}^{t}\right)=\varphi^{-1} \times \varphi^{-1} \circ(\phi \times I d) \circ \kappa_{i}$. Since $(\varphi \times \varphi)^{-1}$ and $\phi \times I d$ are injective and do not contain $p \times p$ in their image the intersection is equal to $(\varphi \times \varphi)_{-1} \circ(\phi \times$ $I d)\left(\mathbb{R}^{2} \times t\right) \cap(\varphi \times \varphi)^{-1} \circ(\phi \times I d)\left(\kappa_{r}(t)\right)$, so we calculate $\left(g_{i}\left(\mathbb{R}^{2} \times t\right) \cap \kappa_{r}(t)\right)$. By our assumption, this is equal to the empty set. Applying $(\varphi \times \varphi)^{-1} \circ(\phi \times I d)$ will give us the desired conclusion.

5. Trivial.

6. Trivial.

7. Since $\operatorname{Im}\left(D_{i}^{t}\right)$ is a subset of the image of $\varphi^{-1} \times \varphi^{-1}$ which is $S^{2} \backslash p \times S^{2} \backslash p$, we get what is needed.

8. Same proof as paragraph (7).

9. Since $\operatorname{Im}\left(D_{i}^{t}\right)=\varphi^{-1} \times \varphi^{-1} \circ(\phi \times I d) \circ \kappa_{i}$, then

$$
\operatorname{Im}\left(D_{i}^{t}\right) \cap \operatorname{Im}\left(D_{j}^{t}\right)=\varphi^{-1} \times \varphi^{-1} \circ(\phi \times I d) \circ \kappa^{i} \cap \varphi^{-1} \times \varphi^{-1} \circ(\phi \times I d) \circ \kappa_{j}
$$

$(\varphi \times \varphi)^{-1}$ and $(\phi \times I d)$ are injective. This is equal to $\varphi^{-1} \times \varphi^{-1} \circ(\phi \times I d)\left(\kappa_{i}(t) \cap\right.$ $\left.\kappa_{j}(t)\right)$. Since $\left(\kappa_{i}(t) \cap \kappa_{j}(t)\right)=\varnothing$ then $\operatorname{Im}\left(D_{i}^{t}\right) \cap \operatorname{Im}\left(D_{j}^{t}\right)$ is also empty.

It is easy to see that for every $j \in J$ the dimension of the manifold we attach to it is smaller than the dimension of $S^{2} \times S^{2}$. Now we will prove that for all $j, k \in J$ if there exist $t \in \mathbb{R}$ such that $\operatorname{Im}\left(r_{t}^{k}\right) \subsetneq \operatorname{Im}\left(r_{j}^{t}\right)$, then $\operatorname{dim}\left(Q_{k}\right)<\operatorname{dim}\left(Q_{j}\right)$ such that $r_{k}$ is the function attached to $k$ and $G_{k}$ is the manifold attached to $k$. We note that if $\operatorname{Im}\left(r_{t}^{k}\right) \subsetneq \operatorname{Im}\left(r_{j}^{t}\right)$ then $\operatorname{Im}\left(r_{t}^{k}\right) \cap \operatorname{Im}\left(r_{j}^{t}\right)=\operatorname{Im}\left(r_{t}^{k}\right)$. Since we know that for any $(i, j) \in$ $J \times J$ and for any $t \in \mathbb{R}$, if we denote the function attached to $k$ as $r_{k}$ we get that $r_{i}^{t} \cap r_{j}^{t}=\underset{k \in f((i, j))}{\bigcup} r_{k}^{t}$. If we have $i, j$ that contradicts our statement there must be $k$ where $k \in f((i, j))$ where $\operatorname{dim}\left(Q_{i}\right)=\operatorname{dim}\left(Q_{j}\right)=\operatorname{dim}\left(Q_{k}\right)$. It is easy to verify that this is not the case.

So by Theorem $4, S^{2} \times S^{2} \backslash\left(\bigcup_{i=1}^{n} \operatorname{Im}\left(G_{i}^{0}\right) \cup \bigcup_{i=1}^{n} \operatorname{Im}\left(D_{i}^{0}\right) \cup\left(p \times S^{2}\right) \cup\left(S^{2} \times p\right) \cup(p \times p)\right)$ is homeomorphic to $S^{2} \times S^{2} \backslash\left(\bigcup_{i=1}^{n} \operatorname{Im}\left(G_{i}^{1}\right) \cup \bigcup_{i=1}^{n} \operatorname{Im}\left(D_{i}^{1}\right) \cup\left(p \times S^{2}\right) \cup\left(S^{2} \times p\right) \cup(p \times p)\right)$. We know that $A \backslash(B \cup C)=(A \backslash C) \backslash(B \backslash C)$. Then it turns out that

$$
S^{2} \times S^{2} \backslash\left(p \times S^{2} \cup S^{2} \times p\right) \backslash\left(\bigcup_{i=1}^{n} \operatorname{Im}\left(G_{i}^{0}\right) \cup \bigcup_{i=1}^{n} \operatorname{Im}\left(D_{i}^{0}\right) \cup p \times S^{2} \cup S^{2} \times p \cup p \times p\right)
$$

is homeomorphic to

$$
S^{2} \times S^{2} \backslash\left(p \times S^{2} \cup S^{2} \times p\right) \backslash\left(\bigcup_{i=1}^{n} \operatorname{Im}\left(G_{i}^{1}\right) \cup \bigcup_{i=1}^{n} \operatorname{Im}\left(D_{i}^{1}\right)\right) \cup p \times S^{2} \cup S^{2} \times p \cup p \times p .
$$

We can see that $S^{2} \times S^{2} \backslash\left(p \times S^{2} \cup S^{2} \times p\right)=S^{2} \backslash p \times S^{2} \times p$. We know from (1) that $\operatorname{Im}\left(G_{j}^{t}\right)=p \times p \cup \phi\left(g_{k} \circ(\varphi \times \varphi)^{-1}\left(\mathbb{R}^{2} \times t\right)\right)$, so if we subtract $p \times p$ we get $\operatorname{Im}\left(G_{j}^{t}\right) \backslash(p \times p)=\left(g_{k} \circ \phi \circ(\varphi \times \varphi)^{-1}\left(\mathbb{R}^{2} \times t\right)\right)$. We can see that the image is inside $\left(\left(S^{2} \backslash p\right) \times\left(S^{2} \backslash p\right) \cup p \times p\right)$ and, therefore,

$$
\varphi \times \varphi\left(\operatorname{Im}\left(G_{j}^{t}\right) \backslash\left(\left(S^{2} \backslash p\right) \times\left(S^{2} \backslash p\right) \cup p \times p\right)\right)=\operatorname{Im}\left(g_{k} \circ \phi\right) .
$$


Additionally, since the image of $D_{i}^{t}$ is inside

$$
\left(\left(S^{2} \backslash p\right) \times\left(S^{2} \backslash p\right) \cup p \times p\right),
$$

$\varphi \times \varphi\left(\operatorname{Im}\left(D_{i}^{t}\right)\right)=\operatorname{Im}\left(\kappa_{i}^{t} \circ \phi\right)$ and since $\varphi \times \varphi$ is a homeomorphism on $S^{2} \backslash p \times S^{2} \times p$, if we apply it we get that $\mathbb{R}^{4} \backslash\left(\bigcup_{i=1}^{n} \operatorname{Im}\left(g_{i}^{0} \circ \phi\right) \cup \bigcup_{i=1}^{n} \operatorname{Im}\left(\kappa_{i}^{0} \circ \phi\right)\right)$ is homeomorphic to $\mathbb{R}^{4} \backslash\left(\bigcup_{i=1}^{n} \operatorname{Im}\left(g_{i}^{1} \circ \phi\right) \cup \bigcup_{i=1}^{n} \operatorname{Im}\left(\kappa_{i}^{1} \circ \phi\right)\right)$. Applying the homeomorphism $\phi$ will give us that $\mathbb{R}^{4} \backslash\left(\bigcup_{i=1}^{n} \operatorname{Im}\left(g_{i}^{0}\right) \cup \bigcup_{i=1}^{n} \operatorname{Im}\left(\kappa_{i}^{0}\right)\right)$ is homeomorphic to $\mathbb{R}^{4} \backslash\left(\bigcup_{i=1}^{n} \operatorname{Im}\left(g_{i}^{1}\right) \cup \bigcup_{i=1}^{n} \operatorname{Im}\left(\kappa_{i}^{1}\right)\right)$, as needed.

Author Contributions: Both authors contributed equally to this paper. Both authors have read and agreed to the published version of the manuscript.

Funding: This research was supported by the ISF-NSFC joint research program (grant No. 3210/19).

Institutional Review Board Statement: Not applicable.

Informed Consent Statement: Not applicable.

Data Availability Statement: Not applicable.

Conflicts of Interest: The authors declare no conflicts of interest.

\section{References}

1. Randell, R. Lattice-isotopic arrangements are topologically isomorphic. Proc. Am. Math. Soc. 1989, 107, 555-559. [CrossRef]

2. Nazir, S.; Yoshinaga, M. On the connectivity of the realization spaces of line arrangements. Ann. Della Sc. Norm. Super. Pisa-Classe Sci. 2012, 11, 921-937.

3. Fan, K.M. Position of singularities and fundamental group of the complement of a union of lines. Proc. Am. Math. Soc. 1996, 124, 3299-3303. [CrossRef]

4. Fan, K.M. Direct product of free groups as the fundamental group of the complement of a union of lines. Mich. Math. J. 1997, 44, 283-291. [CrossRef]

5. Van Kampen, E.R. On the fundamental group of an algebraic curve. Am. J. Math. 1933, 55, 255-260. [CrossRef]

6. Moishezon, B.G.; Teicher, M. Braid Group Technique in Complex Geometry I, Line Arrangements in $\mathbb{C P}$. Contemp. Math. 1988, 78, 425-555.

7. Garber, D.; Teicher, M.; Vishne, U. Classes of wiring diagrams and their invariants. Topology 2003, 42, 265-289. [CrossRef]

8. Garber, D.; Teicher, M.; Vishne, U. $\pi_{1}$-classification of arrangements up to 8 lines. Topology 2003, 42, 265-289. [CrossRef]

9. Ye, F. Classification of moduli spaces of arrangements of 9 projective lines. Pac. J. Math. 2013, 265, 243-256. [CrossRef]

10. Amram, M.; Teicher, M.; Ye, F. Moduli spaces of arrangements of 10 projective lines with quadruple points. Adv. Appl. Math. 2013, 3, 392-418. [CrossRef]

11. Amram, M.; Gong, C.; Teicher, M.; Xu, W. Moduli spaces of arrangements of 11 projective lines with a quintuple point. Turk. J. Math. 2015, 39, 618-644. [CrossRef]

12. Jiang, T.; Yau, S.S.T. Diffeomorphic types of the complements of arrangements of hyperplanes. Compos. Math. 1994, 92, 133-155.

13. Wang, S.; Yau, S.S.T. Rigidity of differentiable structure for new class of line arrangements. Comm. Anal. Geom. 2005, 13, 1057-1075. [CrossRef]

14. Lee, J.M. Introduction to Smooth Manifolds, 2nd ed.; Springer: Berlin, Germany, 2012.

15. Mather, J. Notes on Topological Stability; Harvard University: Cambridge, MA, USA, 1970; Mimeographed Notes. Available online: https://www.maths.ed.ac.uk/ v1ranick/surgery/mather.pdf (accessed on 1 January 2021). 